\title{
Thermal dependence of the properties of cubic boron nitride crystal
}

\author{
Zainelabideen Y. Mijbil ${ }^{1}$, Mudar A. Abdulsattar ${ }^{2}$, Ahmed M. Abdul-Lettif ${ }^{3}$ \\ ${ }^{1}$ Basic Science Department, Veterinary Medicine College, University of Babylon, Hilla, Iraq \\ ${ }^{2}$ Ministry of Sciences and Technology, Baghdad, Iraq \\ ${ }^{3}$ Department of Physics, College of Science, University of Babylon, Hilla, Iraq; *Corresponding author: abdullettif@yahoo.com
}

Received 20 November 2010; revised 23 December 2010; accepted 26 December 2010.

\begin{abstract}
Lattice constant, total energy, cohesive energy, bulk modulus, speed of sound (U), plasmon energy $\left(E_{p l}\right)$, valence charge distribution and energy bands of cubic boron nitride crystal have been calculated and studied as a function of temperature using self-consistent field tight binding method with complete neglect of differential overlap version 2 using 8-atom large unit cell approach. Our results illustrate that the increase of temperature leads to an increase of lattice constant, cohesive energy, and valence charge distribution at the atoms, whereas a decrease is obtained for bulk modulus, energy band widths, valence charge distribution in the intratomic distance, speed of sound, and the plasmon energy. The comparison with experimental and other theoretical results has showed an excellent agreement for the lattice constant, bulk modulus, cohesive energy, speed of sound, plasmon energy value and the valence band, whereas remarkable differences in charge distribution values, and the band gap are found. These differences are common in the results that depend on this type of calculation. Values for conduction band and speed of sound have not been found for comparison. Our relation for $E_{p r} T$ fails but the $U-T$ relation is successful.
\end{abstract}

Keywords: Electronic Structure; Temperature Dependence; Boron Nitride

\section{INTRODUCTION}

The high account of hardness [1], melting point [2], high thermal conductivity [3] and band gap [4], resistance for oxidation as temperature increases [5] with inert state [6] low electrical conductivity [7], and no iron reaction [8] are all for $s p^{3}$ [9] cubic boron nitride. So it has amazing aspects [10] of multiuse [11,12] such as in polishing, cutting [13] and protection [14], and it has been the core for many studies [15-18] since its discovery in 1957 [19].

Methods of semiempirical calculations give a privilege of reasonable computer execution time for getting the results due to the approximations which are involved $[20,21]$, accordingly they are used in many studies [22-25]. In this paper we have used linear combination of atomic orbitals (LCAO) approach as a starting point [26] which includes both 1) the CNDO, as one of the primary semiempirical methods, that is presented by Popel et al. [27] in which many electron-electron interactions had been neglected. CNDO focused on both valence electrons and nuclei as a core of the atom (with the other electrons) [21], and 2) LUC (Large Unit Cell) approximation or (Supercell) [28] is used in order to minimize the size of Brillion Zone (BZ) and so to reduce the number of the wave vector $(\mathrm{k})$ points in which the aspects of the crystal such as band structure could be calculated $[29,30]$.

The aim of this project is to study the c-BN by $\mathrm{CNDO} / 2$. $\mathrm{CNDO} / 2$ is an old fashion method but here we try to see its merits and drawbacks through works and results due to the trend to couple between ab initio calculations with semiempirical methods, specially if we know that the ab initio method is used for small systems, in comparison with the semiempirical calculations which were applied for large systems, so it is a competition between the accuracy of the former and the high speed of the later [31].

\section{METHODOLOGY}

According to LCAO-LUC with eight atoms, and STO (Slater Type Orbital), the atomic orbital is of the form:

$$
\Psi(r, \theta, \varphi)=G r^{n^{*}-1} \exp (-r \xi) Y(\theta, \varphi)
$$

where $\Psi(r, \theta, \varphi)$ is Slater type orbital. $r, \theta, \varphi$ are the polar spherical coordinates of the atom. $G$ is the normalization 
constant. $n^{*}$ is the effective principal quantum number, and $Y(\theta, \varphi)$ is the spherical harmonics [32]. The Fockmatrix element $\left(F_{\mu \mu}\right)$ and the total energy $(E)$ can be calculated as [21]:

$$
\begin{gathered}
F_{\mu \mu}=-\frac{1}{2}\left(I_{\mu}+A_{\mu}\right)+\left[0.5\left(1-P_{\mu \mu}\right)-Q_{A}\right] \gamma_{A A}-\sum_{B(\neq A)} Q_{B} \gamma_{A B} \\
F_{\mu \nu}=\beta_{A B} S_{\mu \nu}-0.5 P_{\mu \nu} \gamma_{A B} \\
E=0.5 \sum_{\mu \nu}\left[P_{\mu \nu}\left(H_{\mu \nu}-F_{\mu \nu}\right)\right]+\sum_{A<B} Z_{A} Z_{B} R_{A B}^{-1}
\end{gathered}
$$

where $\mu$ and $v$ represent atomic orbitals and here they are used either as $s$ or $p$ orbitals, the quantity $-0.5\left(I_{\mu}+A_{\mu}\right)$ is either EES or EEP for $\mu \equiv s$ or $p$ respectively, $I_{\mu}$ and $A_{\mu}$ represent the ionization potential and the atomic electron affinity respectively, $\beta_{A B}$ is the bonding parameter, $P_{\mu \mu}$ is the density matrix, $Q_{B}$ and $Q_{A}$ are the net charge on atom $A$ and $B$ respectively, and $\gamma_{A B}$ is the average electrostatic repulsion between any electron on atom $\mathrm{A}$ and any electron on atom $B$.

The final expressions with LUC approaches [20] are:

$$
\begin{aligned}
& F_{\mu \mu}(0)=-\frac{1}{2}\left(I_{\mu}+A_{\mu}\right)+0.5 \gamma_{A A}^{o o}-\sum_{B} Q_{B}(0) \sum_{z} \gamma_{A B}^{o z} \\
& +\sum_{z \neq o} \beta_{A B}^{o z} S_{o \mu, z v}-0.5 \sum_{z} P_{\mu \mu}(0) \gamma_{A A}^{o z} f(x) \\
& F_{\mu \nu}(0)=\sum_{z} \beta_{A B}^{o z} S_{o \mu, z v}-0.5 P_{\mu \nu}(0) \sum_{v} \gamma_{A B}^{o z} f(x) \\
& E=0.5 \sum_{\mu v} P_{\mu \nu}(0)\left[F_{\mu v}(0)+H_{\mu v}(0)\right] \\
& \quad+0.5 \sum_{A} \sum_{B} Z_{A} Z_{B} R_{A B}^{-1}
\end{aligned}
$$

where $f(x)$ it is Szymanski enhancement function, $o$ is the central LUC and $z$ is the other LUCs [33].

We have firstly studied how $\mathrm{CNDO} / 2$ parameters: the orbital exponent $(\xi)$, bonding parameter $(\beta), s$-shell electronigativity $(E E S)$ and $p$-shell electronegativity $(E E P)$ could be changed and how this change may affect the results. Our suggested parameters are shown in Table 1, and secondly we have calculated the lattice constant, cohesive energy, valence band width, conduction band width, direct band gap, according to these parameters as shown later.

Table 1.The parameters of $\mathrm{CNDO} / 2$.

\begin{tabular}{cccccccccc}
\hline Property & \multicolumn{2}{c}{$-\beta(\mathrm{eV})$} & \multicolumn{2}{c}{$\xi$ (a.u.) } & \multicolumn{2}{c}{$E E S(\mathrm{eV})$} & \multicolumn{2}{c}{$E E P(\mathrm{eV})$} \\
\hline Reference & $\mathrm{B}$ & $\mathrm{N}$ & $\mathrm{B}$ & $\mathrm{N}$ & $\mathrm{B}$ & $\mathrm{N}$ & $\mathrm{B}$ & $\mathrm{N}$ \\
{$[33]$} & 7.452 & 13.95 & 1.531 & 1.957 & 5 & 9.158 & 4.128 & 7.511 \\
{$[21]$} & 17 & 25 & 1.3 & 1.95 & 9.549 & 19.316 & 4.001 & 7.375 \\
Pres. & 8.455 & 11.95 & 1.53 & 1.95 & 28.416 & 34.766 & 14.666 & 22.728 \\
\hline
\end{tabular}

The procedure of the present work can be summarized as follows:

1) We change the four parameters of the CNDO method $\xi, \beta_{A B}$, EES and EEP in arbitrary way until we get a value of the lattice that is very close to the experimental value with the minimum value of the total energy of the crystal.

2) Then we measure the values of the cohesive energy and all the energies of the electronic band.

3 ) If the values of energies are in agreement with the experiments then the iterations are ended and we fix the parameters.

4) If the values of the energies are incorrect the parameters are changed until getting the closest values to the experiments.

\section{CALCULATIONS}

\subsection{The Total Energy}

The total energy $(E)$ is calculated when the difference between two successive iterations from the program is less than $(0.0136 \mathrm{eV})$ and our result is fitted to BirchMurnaghan equation [34] as below:

$$
E=E_{o}+\frac{B_{o} V}{B_{o}^{\prime}}\left[\frac{\left(V_{o} / V\right)^{B_{o}^{\prime}}}{B_{o}^{\prime}-1}+1\right]-\frac{B_{o} V_{o}}{B_{o}^{\prime}-1}
$$

where $E_{o}, V_{o}$, and $B_{o}$ are the total energy, volume of the unit cell and the bulk modulus at the equilibrium point, zero temperature-pressure point, respectively, $V$ is the volume at any temperature, and $B_{o}^{\prime}$ is the pressure derivative of bulk modulus with a value of (3.79) [35].

\subsection{Cohesive Energy}

The cohesive energy $\left(E_{c o h}\right)$ is calculated from the LUC total energy according to the equation [36]:

$$
E_{c o h}=\frac{E}{8}-E_{\text {free }}-E_{z p}
$$

$E_{\text {free }}$ is the energy of ( $s p$ ) orbitals for the free atom and equals to $169.164 \mathrm{eV}$ [37], and $E_{z p}$ is the zero point energy and equals to $0.165 \mathrm{eV}$ [38].

\subsection{Volume}

For tetrahedral structure it is found that [39]:

$$
\alpha \times T_{m} \cong 0.021
$$

$\alpha$ is the linear thermal expansion and $T_{m}$ is the melting temperature [39] which equals to $3000 \mathrm{~K}$ [40]. We have also from [40] that:

$$
\alpha=\frac{1}{a_{o}} \frac{\partial a}{\partial T}
$$


$T$ is the temperature, $a$ is the lattice constant at any temperature and $a_{o}$ is the lattice constant at zero temperature. If we take the initial temperature equals to $(0 \mathrm{~K})$, and combine Eq.10 to Eq.11 we get a relation for tetrahedral structure:

$$
a=a_{o}\left[1+0.021 \frac{T}{T_{m}}\right]
$$

Let $\left[1+0.021 T / T_{m}\right] \equiv X$, and we have $V=a^{3}$ then we will get:

$$
V=V_{o} X^{3}
$$

\subsection{Bulk Modulus}

The bulk modulus has been calculated from the Cohen empirical formula [41]:

$$
B_{o}=\frac{1970-200 I^{C}}{d^{3.5}}
$$

$I^{C}$ is the Cohen ionicity factor (= 0,1 and 2 for IV, III-V and II-V groups respectively) [35], and it is used instead of Pauling ionicity factor $\left(I^{P}\right)(\mathbf{E q . 1 5}[42]), d(=0.433 a)$ is the intratomic distance [43].

$$
I^{P}=1-\exp \left[-\frac{\left(\chi_{A}-\chi_{B}\right)^{2}}{4}\right]
$$

where $\chi_{A}$ and $\chi_{B}$ are the atom $A$ and $B$ electronigativity respectively.

Now by substituting Eq.13 in Eq.14 with some simple mathematical treatment, we get a general relation for tetrahedral structure:

$$
B=B_{o} X^{-3.5}
$$

\subsection{Plasmon Energy and the Speed of Sound}

According to (Kumar and Sastry) [40] the plasmon energy $\left(E_{p l}\right)$ is defined as:

$$
d=15.3 E_{p l}^{-\frac{2}{3}}
$$

From the above relations we have $d=d_{o} X$, so the general relation for tetrahedral structure is:

$$
E_{p l}=\left[\frac{d_{o}}{15.3} X\right]^{-\frac{3}{2}}
$$

For the speed of sound $(v)$ we have [44]:

$$
v=\sqrt{\frac{B}{\rho}}
$$

$\rho$ represents the density at any temperature and relates to $\rho_{o}(=3.47 \mathrm{~g} / \mathrm{cm}$ according to [45] $)$ the density at zero temperature with the relation:

$$
\rho=\rho_{o} \frac{V_{o}}{V}
$$

So by gathering Eqs.13,16,19,20 we get:

$$
\begin{gathered}
v=v_{o} X^{-0.25} \\
v_{o}=\sqrt{\frac{B_{o}}{\rho_{o}}}
\end{gathered}
$$

\subsection{Energy Bands}

They are found by calculating the energy difference between the symmetric points where $\left(\Gamma_{25 \mathrm{~V}}-\Gamma_{1 \mathrm{~V}}\right)$ determines the valence band $[46,47],\left(\mathrm{X}_{1 \mathrm{C}}-\Gamma_{25 \mathrm{~V}}\right)$ for indirect band gap, $\left(\Gamma_{15 \mathrm{C}}-\Gamma_{25 \mathrm{~V}}\right)$ for direct band gap $[1,33]$, while $\left(\mathrm{X}_{5 \mathrm{C}}-\mathrm{X}_{1 \mathrm{C}}\right)$ for conduction band [48].

\subsection{Valence Charge Density}

The electronic charge distribution $\rho(r)$ can be calcu-

\begin{tabular}{|c|c|c|c|c|c|c|c|}
\hline $\begin{array}{l}\text { Prop- } \\
\text { erty }\end{array}$ & Pres. & Exp. & [33] & [1] & [49] & [38] & {$[43]$} \\
\hline$a_{o}(\mathrm{~A})$ & 3.6192 & $3.617[50]$ & 3.6 & 3.606 & 3.623 & 3.63 & 3.582 \\
\hline $\begin{array}{c}B_{o} \\
(\mathrm{GPa})\end{array}$ & 367.73 & 368 [50] & 1132 & 367 & 365.3 & 360 & 392.3 \\
\hline$B_{o}^{\prime}$ & 4.848 & & & & 3.94 & & 3.763 \\
\hline $\begin{array}{l}E_{c o h} \\
(\mathrm{eV})\end{array}$ & 13.21 & $13.2[1]$ & 8.131 & 14.3 & 6.7117 & 13.9 & \\
\hline $\begin{array}{l}\mathrm{DBG} \\
(\mathrm{eV})\end{array}$ & 23.929 & $14.5[51]$ & 16.01 & 8.6 & & & \\
\hline $\begin{array}{l}\text { VBW } \\
(\mathrm{eV})\end{array}$ & 20.121 & $20.6[51]$ & 21.78 & 20.3 & & & \\
\hline $\begin{array}{l}\mathrm{CBW} \\
(\mathrm{eV})\end{array}$ & 14.05 & & & & & & \\
\hline$\eta$ & 5.019 & & & & & & \\
\hline$v(\mathrm{~m} / \mathrm{s})$ & 10326.813 & & & & & & \\
\hline$E_{p l}(\mathrm{eV})$ & 30.50366167 & 22.758 [52] & $\begin{array}{c}24.53 \\
{[52]}\end{array}$ & $\begin{array}{c}30.55 \\
{[40]}\end{array}$ & & & \\
\hline
\end{tabular}
lated at any point from the equation:

$$
\rho(r)=\sum_{\mu \nu} P_{\mu \nu} \phi_{\mu}(r) \phi_{\nu}(r)
$$

$P_{\mu \nu}$ is the density matrix, and both $\emptyset_{\mu}(r)$ and $\emptyset_{\nu}(r)$ are the basis functions.

\section{RESULTS AND DISCUSSION}

The calculated properties of c-BN at $300 \mathrm{~K}$ and $0 \mathrm{GPa}$ are illustrated in Table 2.

Table 2. The structural and electronic properties of c-BN at $300 \mathrm{~K}$ and $0 \mathrm{GPa}$. 


\subsection{Total Energy}

Figure 1 shows the curve of the total energy against the lattice constant, and from it we can see that when atoms come closer, the attractive and repulsive forces appear [53] so the atomic orbitals overlap to make bonds [54]. The overlap presents three states:

1) If $a>a_{o}$; the attractive force $\left(\mathrm{F}_{\mathrm{a}}\right)$ will be grater than the repulsive force $\left(\mathrm{F}_{\mathrm{r}}\right)$ due to the existence of the electrons in the region between the atoms.

2) If $a<a_{o} ; \mathrm{F}_{\mathrm{a}}<\mathrm{F}_{\mathrm{r}}$, this case is due to the electronelectron and nuclei-nuclei repulsions, in addition to Pauli exclusion principle which prevents atoms to be closer than $a_{0}$.

3) If $a=a_{o} ; \mathrm{F}_{\mathrm{a}}=\mathrm{F}_{\mathrm{r}}$, which is the balance case.

\subsection{Cohesive Energy}

According to Eq.9 the cohesive energy mainly depends upon the total energy and when the distance increases by temperature the balance state will be shifted therefore the cohesive energy increases as shown in Figure 2.

\subsection{Volume}

The relation between the volume and temperature, as shown in Figure 3, can be simply attributed to the fact that the atom interacts mainly with its neighbor [55]

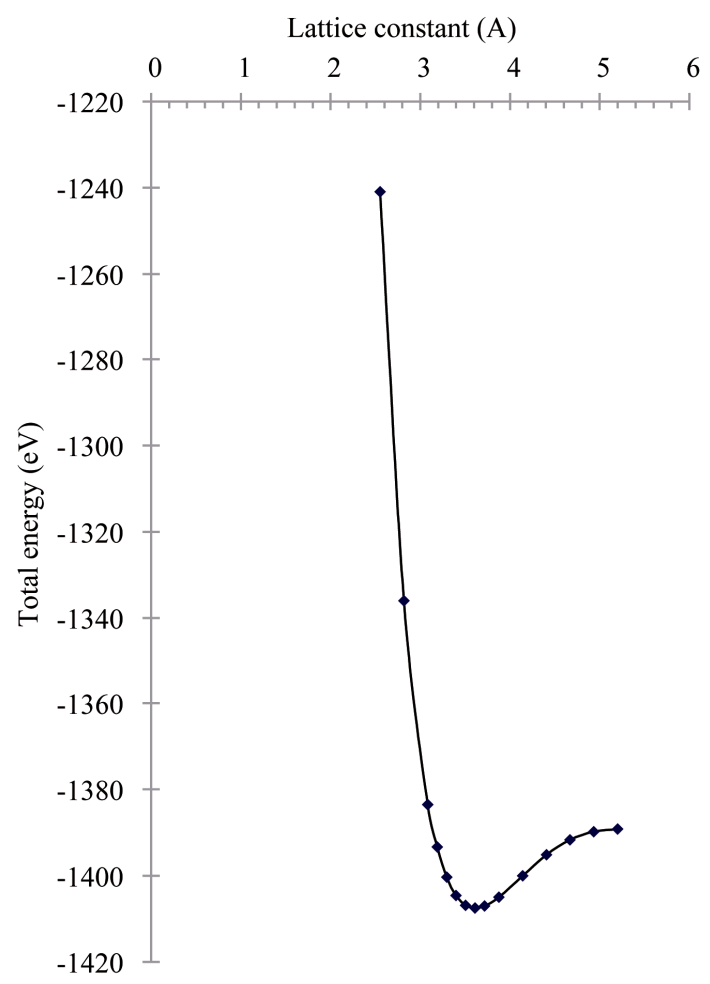

Figure 1. Variation of the total energy with the lattice constant.

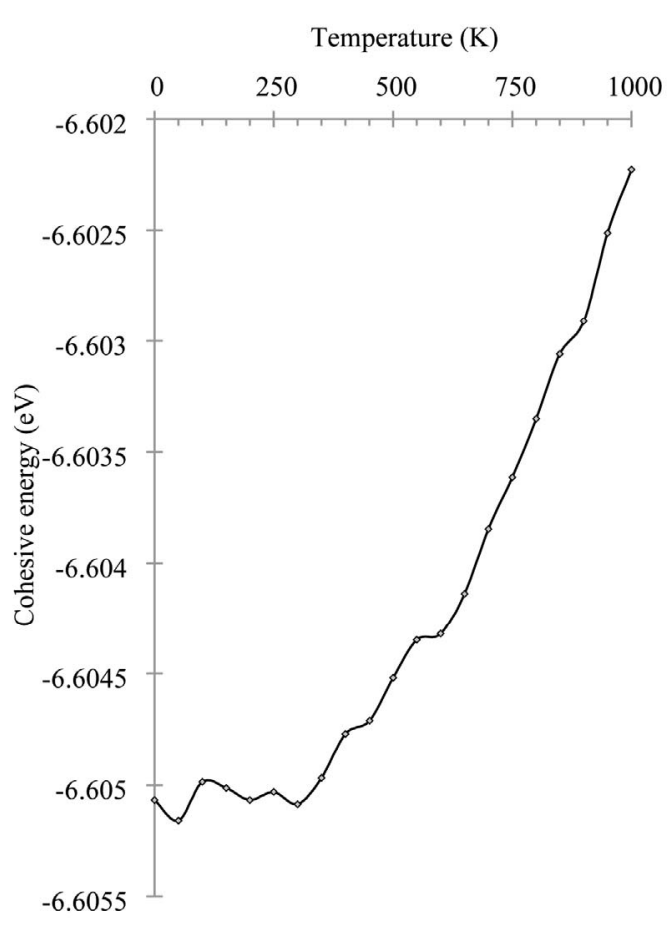

Figure 2. Effect of temperature on the cohesive energy.

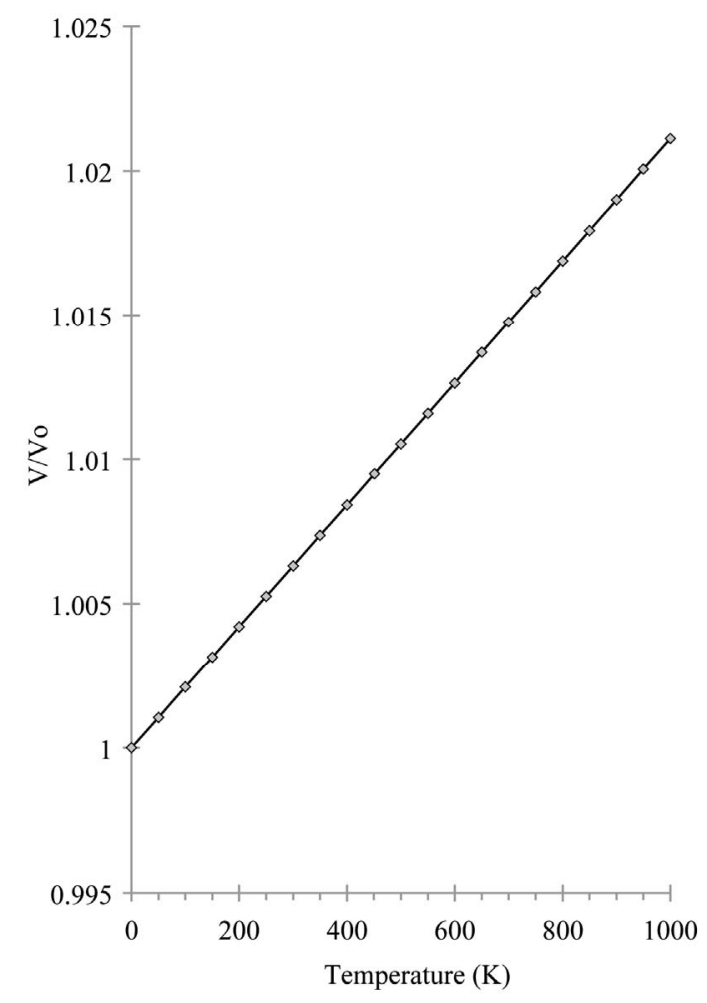

Figure 3. Effect of temperature on the volume.

In the meanwhile, when temperature is increased the kinetic rgy $\left(E_{k}\right)$ also increases according to [56]: 


$$
E_{k}=\frac{3}{2} k_{B} T
$$

Depending on Born-von Kármán theory which considers the lattice as a punch of coupled harmonic oscillators [57]. We have from this view [58]:

$$
E_{k}=\frac{1}{2} C\left\langle u^{2}\right\rangle
$$

where $C$ is force constant, $\left\langle u^{2}\right\rangle$ is the mean square displacement of the atom, so by combing the last two equations we get:

$$
\left\langle u^{2}\right\rangle=\frac{3 k_{B}}{C} T
$$

The latter equation means that the increase in temperature entails an increase in atomic vibration amplitude which makes the bond weaker [59] and the volume will increase.

\subsection{Bulk Modulus}

Bulk modulus represents the hardness [60] and determines the ability of matter to withstand the volume change [43], so it depends upon the bond length [4] and this bond is short and very strong in c-BN [61]. Temperature increases volume and kinetic energy [56] which entails a decrease in density, resistance to volume change and bond strength [62] which decreases the bulk modulus [63] as shown in Figure 4 because it depends upon the density directly [64]. This behavior is noticed for silicon [65], diamond [55], and also for other solids [66-70].

\subsection{Plasmon Energy and Speed of Sound}

Here we have presented two relations: one of them is for plasmon energy and the other is for speed of sound with temperature in order to simplify the calculations. Our results for plasmon energy as in Figure 5 are in contrast with the experiment $[71,72]$ because we have depended on a relation that does not take into account the effective ionic charge $e_{T}^{*}$ which is directly related to the plasmon energy [73]:

$$
E_{p l}=e_{T}^{*}\left(\frac{4 \pi n}{M \varepsilon_{\infty}}\right)^{\frac{1}{2}}
$$

where $n$ is the charge density [74], $M$ is the reduced mass of the two atoms and $\varepsilon_{\infty}$ is the infrared dielectric constant. Experimentally [71] and theoretically [75] $e^{*}{ }_{T}$ increases with $d$ and vice verse, so the plasmon energy must also increase with $d$. As a result this equation fails.

On the other hand the speed of sound as in Figure 6,

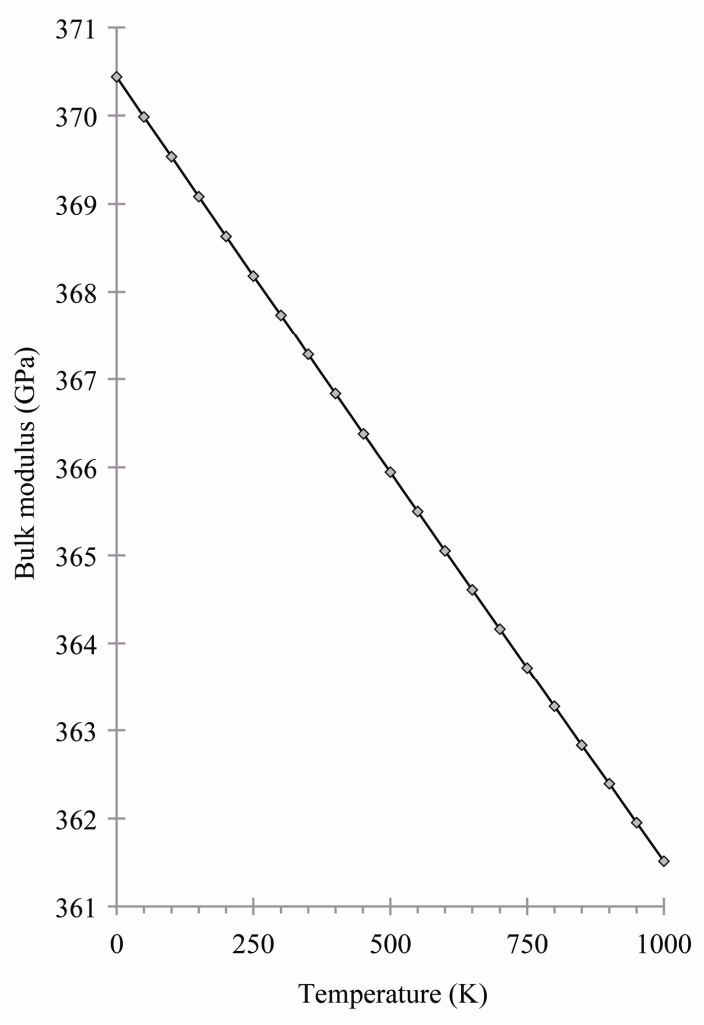

Figure 4. Effect of temperature on the bulk modulus.

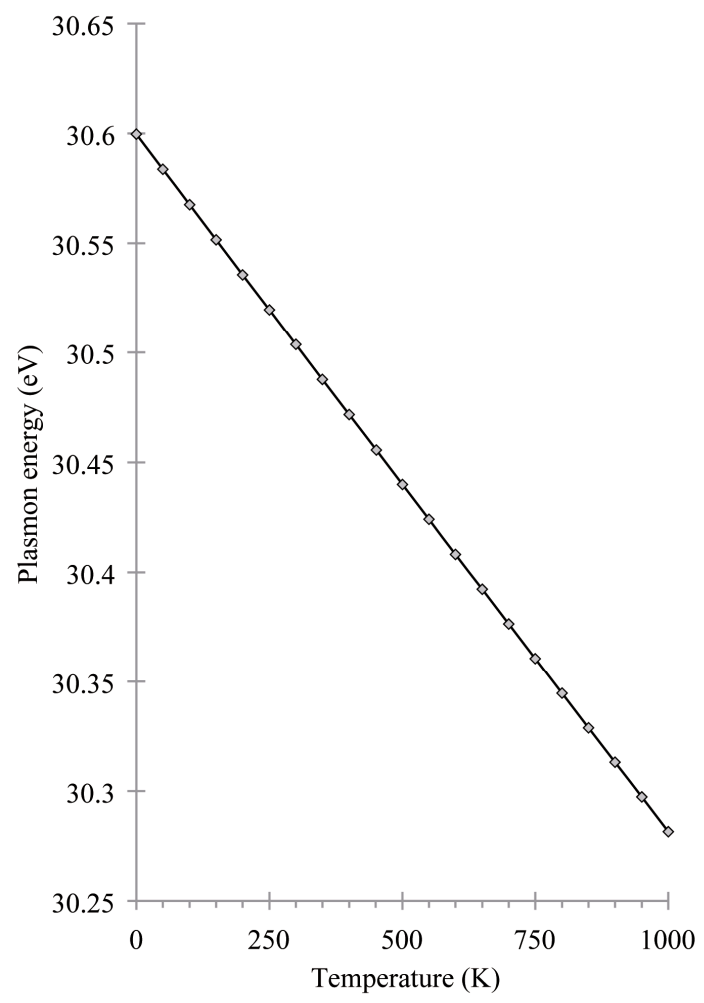

Figure 5. Effect of temperature on the plasmon energy. 


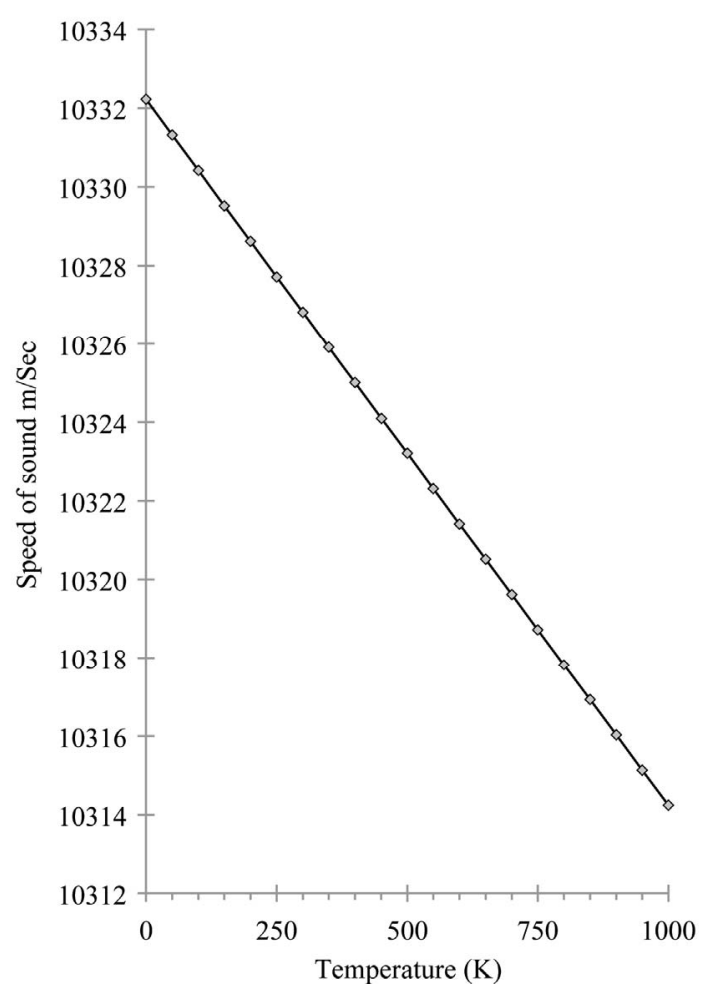

Figure 6. Effect of temperature on the speed of sound.

which also represents the average of phonon velocity [76] and is related to the average energy of phonon $\bar{E}_{p h}$ by $\mathbf{E q}$. 28, behaves consistently with other experimental calculations [71] for III-V compounds and theoretical calculations for InP [44,72], Si and Diamond [77]

$$
\bar{E}_{p h}=\hbar v\left(\frac{6 \pi N}{V}\right)^{\frac{1}{3}}
$$

\subsection{Energy Bands}

The energy bands show the following points:

1) The value of direct band gap is greater than the experimental value as shown in Table 3. This behavior is also noticed in most calculations that use Hartree-Fock method [78-80] due to its approximations which are:

- The minimal basis sets include ( $s$ and $p$ ) orbitals only, these orbitals determine the valence and the conduction band accurately, while the outer orbitals that have not been taken into account decrease the value of the band gap [81].

- We have not considered the correlation corrections which decrease the value of the indirect band gap [79].

- Both $s$ and $p$ orbitals are given the same value of $\xi$ and $\beta$ and the latter parameter determines the bonding and the antibonding states.

- Neglecting of core states which affect the outer va- lence electrons distribution [36].

2) All bands decrease with temperature as shown in Figures 7 and 8.

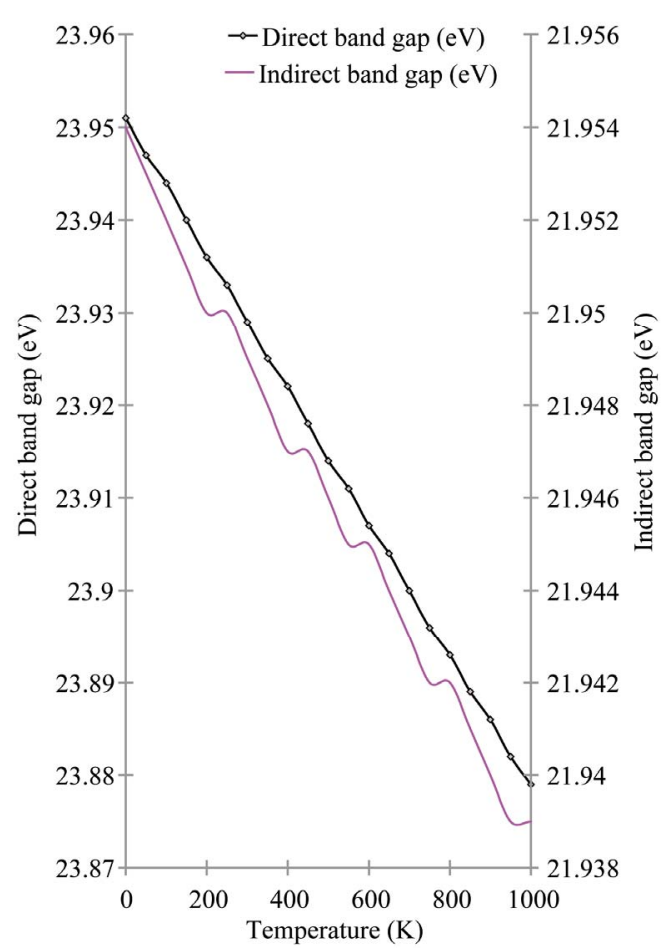

Figure 7. Effect of temperature on the direct and indirect band gaps.

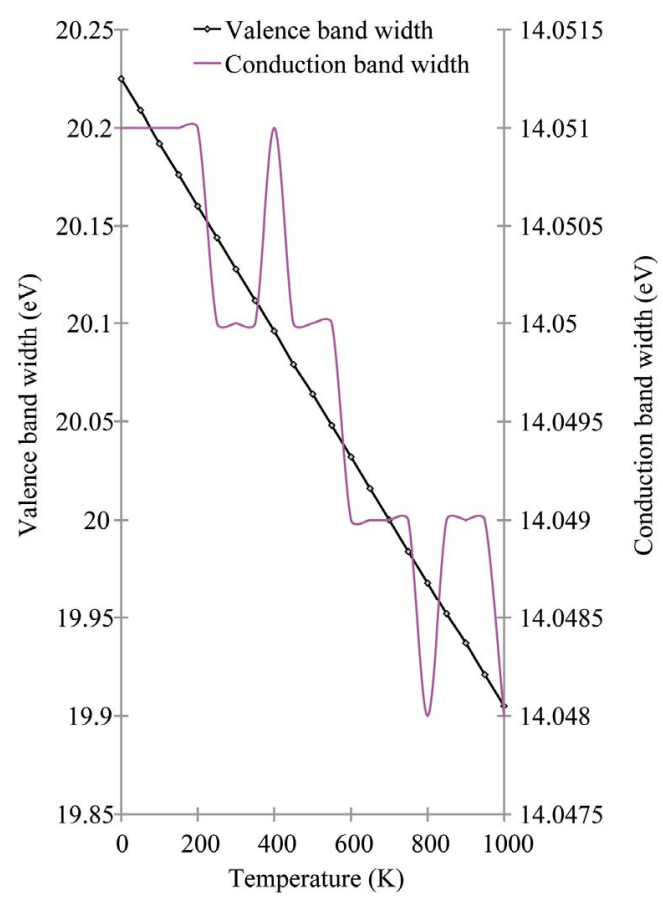

Figure 8. Effect of temperature on valence and conduction bands. 
Table 3. The symmetry points value with their approximate structure in $(\mathrm{eV})$.

\begin{tabular}{lllll}
\hline Points & $\begin{array}{l}\text { Approximate } \\
\text { structure }\end{array}$ & Pres. & {$[33]$} & Exp. [33] \\
\hline$\Gamma_{1 \mathrm{~V}}$ & $\begin{array}{l}\text { Nitrogen bonding } s \\
\text { orbitals }\end{array}$ & -20.1218 & -21.78 & -20.06 \\
$\Gamma_{1 \mathrm{C}}$ & $\begin{array}{l}\text { Boron antibonding } \\
s \text { orbitals }\end{array}$ & 35.792 & 16.38 & \\
$\Gamma_{15 \mathrm{~V}}$ & $\begin{array}{l}\text { Nitrogen bonding } \\
p \text { orbitals }\end{array}$ & 0 & 0 & 0 \\
$\Gamma_{15 \mathrm{C}}$ & $\begin{array}{l}\text { Boron antibonding } \\
p \text { orbitals }\end{array}$ & 23.932 & 16.06 & 8.19 \\
$\mathrm{X}_{1 \mathrm{~V}}$ & $\begin{array}{l}\text { Nitrogen bonding } s \\
\text { orbitals }\end{array}$ & -13.0319 & -10.88 & \\
$\mathrm{X}_{1 \mathrm{C}}$ & $\begin{array}{l}\text { Boron antibonding } \\
s \text { orbitals }\end{array}$ & 21.951 & 12.34 & 6 \\
\hline
\end{tabular}

Symmetric points are a combination of atomic orbitals to produce either bonding or antibonding orbitals [82]. Our calculations of the main points with their relativistic corrections are illustrated in Table $\mathbf{3}$ and compared with (Zunger and Freeman) [10] and Ferhat et al. [42] who both explained the structure of these points.

Valence band is nearly determined by $(2 s$ and $2 p)$ nitrogen bonding orbitals [82], while the conduction band is determined by $(2 s$ and $2 p$ ) boron antibonding orbitals $[79,40]$. Therefore, when the difference between these points increases, the energy bands will be wider. Our results are consistent with $[1,10,83]$ for $\mathrm{c}-\mathrm{BN}$.

The effect of temperature is similar to the effect of tension pressure but it is thermal [84], while it directly affects the band gaps [85]. Due to the increase in tem- perature which leads to increase in distance, the orbitals overlap will be decreased and the repulsion will also decrease, and the energy difference between bonding and antibonding states will be decreased accordingly. This means that the widths become narrower. Our results are consistent with Pässler's results for semicoductors [86], and with measurements for band gab as reported by Olguín et al. [87] and Fan [88].

\subsection{Valence Charge Distribution}

First of all we need to mention the approximations used in CNDO method because this kind of results is sensitive to them, and they are:

1) Zero differential overlap which neglects the repulsion integrals and the electron-electron overlap on the same atom, and these change the Coulomb potentials that affect the distribution [89].

2) The values of EES and EEP are very high, "two of the semiempirical method parameters used to get the results", and both of them represent the electronigativity of the atom and determine the bond polarity [90].

3) Correlation corrections have not been taken into account.

Figures 9-11 and the high anharmonicity parameter $(\eta)$ [91] in Table 2 show that the density of the charge is higher around nitrogen's atoms than boron's atoms, because the electrons can stay near one of them more than the another which leads to transfer of charge which is called charge transfer factor $\left(\mathrm{c}_{\mathrm{i}}\right)$. This dimensionless parameter reflects the ability of atom to lose electrons either for another atom or for the intratomic distance.

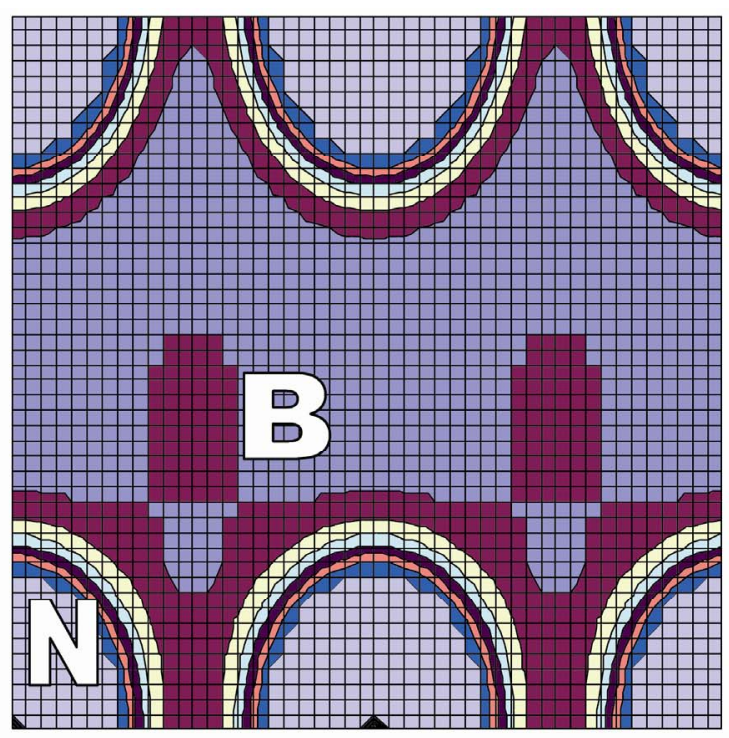

(a)

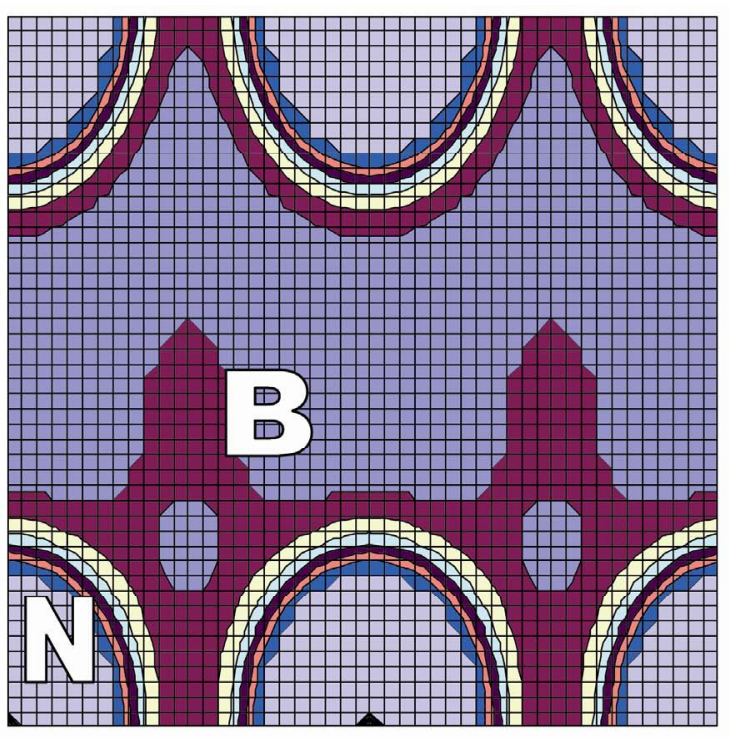

(b)

Figure 9. Valence charge distribution of the plane (110): a - at (0K) and b - at (1000K). 


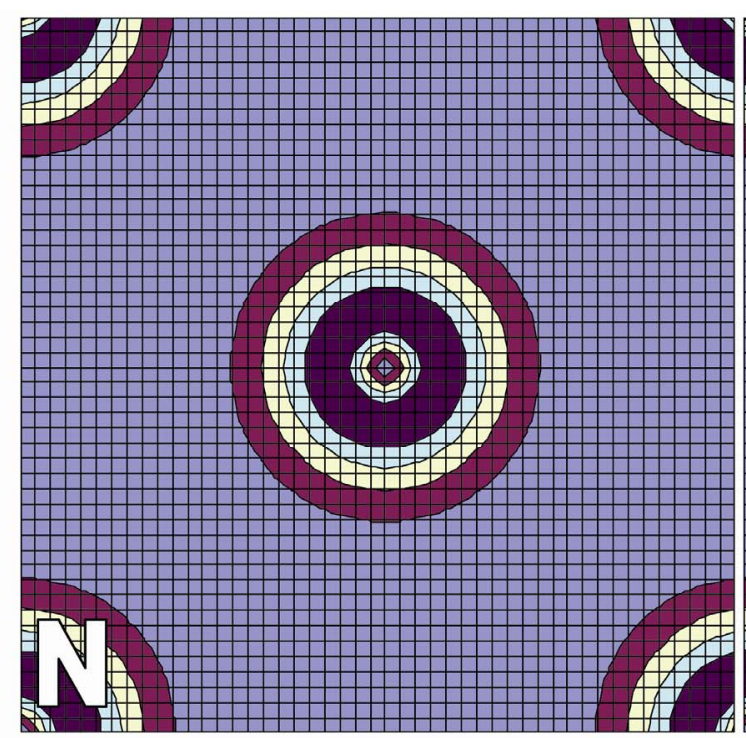

(a)

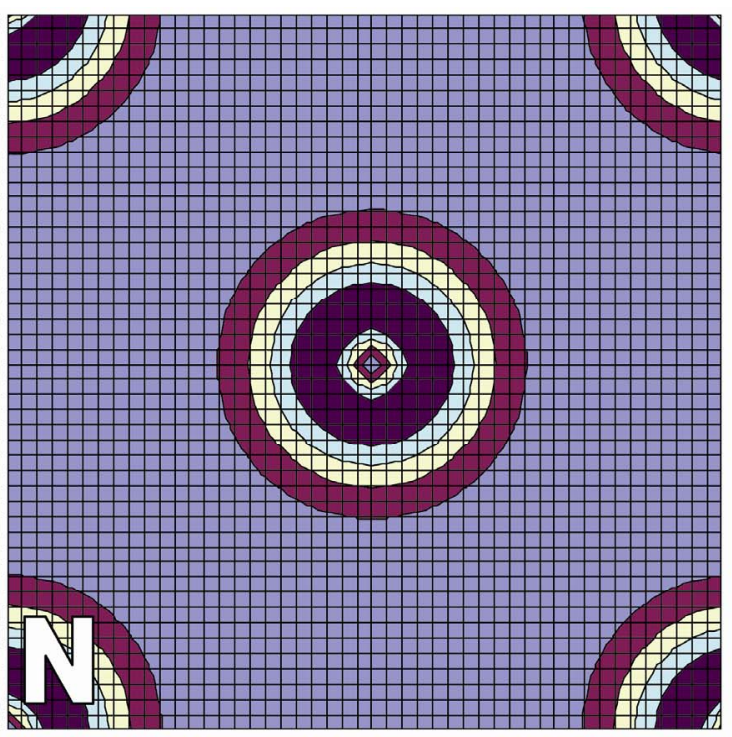

(b)

Figure 10. Valence charge distribution of the plane (001): a - at (0K) and b - at (1000K).

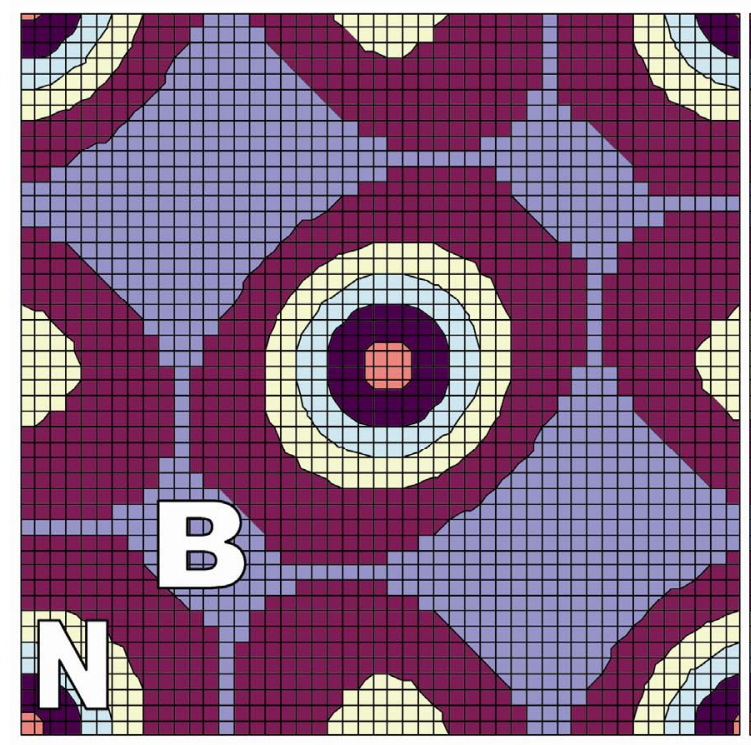

(a)

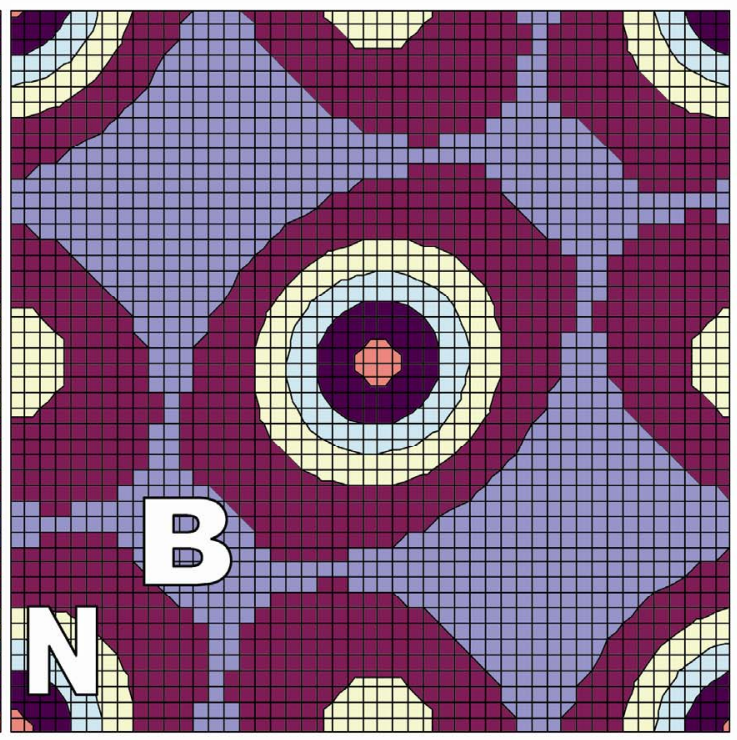

(b)

Figure 11. Valence charge distribution of the plane (400): a - at (0K) and b - at (1000K).

This case is obvious in polar compounds such as c-BN which has $\left(c_{i}=0.8\right)$ [92]. On the other hand, nitrogen pulls the electrons from boron [93] due to its high electronigativity with respect to boron [94], but as temperature increases the atoms try to separate and return to their original state, as free atoms [95].

\section{CONCLUSIONS}

1) TB-LUC-CNDO/2 method is preferred for wide range of calculations such as the lattice constant, cohesive energy and valence band etc..
2) In this method the energy bands can not be accurately calculated all together.

3) The correlation corrections have remarkable effects upon the band gaps' width and charge distribution.

4) The c-BN tends to be more conductive with temperature increase.

5) Even with high temperature, c-BN can withstand corrosions and abrasives.

6) The infrared absorption of c-BN increases with the increase of temperature. 


\section{REFERENCES}

[1] Wentzcovitch, R.M., Chang, K.J. and Cohen, M.L. (1986) Physical Review B, 34, 1071-1079. doi:10.1103/PhysRevB.34.1071

[2] Sun, H., Jhi, S.H., Roundy, D., Cohen, M.L., Louie, S.G. (2001) Physical Review B, 64, 094108/1-6.

[3] Kádas, K., Kern, G. and Hafner, J. (2000) Surface Science, 494-497. doi:10.1016/S0039-6028(00)00254-5

[4] Mattesini, M. and Matar, S.F. (2001) Computational Materials Science, 20, 107-119. doi:10.1016/S0927-0256(00)00132-4

[5] Major, B., Mróz, W., Jelinek, M., Kosydar, R., Kot, M., Major, Ł., Burdyńska, S. and Kustosz, R. (2006) Bulletin of the Polish Academy of Sciences: Technical Sciences, 54, pp. 181-188.

[6] Knittle, E., Kaner, R.B. Jeanloz, R. and Cohen, M. L. (1995) Physical Review B, 51, 12149-12156. doi:10.1103/PhysRevB.51.12149

[7] Lambrecht, W.R.L. and Segall, B. (1989) Physical Review $B$, 40, 9909-9919.

[8] Watanabe, S., Wheeler, D.R., Abel, P.B., Murakawa, M. and Miyake, S. (1998) Surface chemistry, microstructure, and tribological properties of cubic boron nitride films. NASA Lewis Research Center.

[9] Mãrlid, B. (2001) Theoretical modling of thin film growth in the B-N system. Acta Universitais.

[10] Zunger, A. and Freeman, A.J. (1978) Physical Review B, 17, 2030-2042. doi:10.1103/PhysRevB.17.2030

[11] Meng, Y., Mao, H., Eng, P.J., Trainor, T.P., Newville, M., Hu, M.Y., Kao, C., Shu, J., Hausermann, D. and Hemley, R. J. (2004) Nature Materials, 3, 111-114. doi:10.1038/nmat1060

[12] Karlsson, J. and Larsson, K. (2010) Journal of Physical Chemistry C, 114, 3516-3521. doi:10.1021/jp907186a

[13] Horiuchi, S., Huang, J.Y., He, L.L., Mao, J.F. (1998) Philosophical Magazine A, 78, 1065-1072. doi:10.1080/01418619808239974

[14] Huang, J.Y. and Zhu, Y.T. (2002) Chemistry of Materials, 14, 1873-1878. doi:10.1021/cm0109645

[15] Hang-Sheng, Y., Fa-Min, Q. and An-Min, N. (2010) Journal of Inorganic Materials, 25, 748-752. doi:10.3724/SP.J.1077.2010.00748

[16] Khavryuchenko, O.V., Alekseev, S.A., Beobide, A.S., Kandilioti, G., Voyiatzis, G.A., Lisnyak, V.V. (2010) The Journal of Physical Chemistry C, 114, 1102-1109. doi:10.1021/jp908711x

[17] Zhao, Y. and Wang, M. (2009) Journal of Materials Processing Technology, 209, 355-359. doi:10.1016/j.jmatprotec.2008.02.005

[18] Yallese, M.A., Chaoui, K., Zeghib, N., Boulanouar, L., Rigal, J.F., (2009) Journal of Materials Processing Technology, 209, 1092-1104. doi:10.1016/i.jmatprotec.2008.03.014

[19] Wentorf, R.H., Jr. (1962) Journal of Chemical Physics, 34, 809-812. doi:10.1063/1.1731679

[20] Harker, A.H. and Larkins, F.P. (1979) Journal of Physics C: Solid State Physics, 12, 2487-2495. doi:10.1088/0022-3719/12/13/013

[21] Pople, J.A. and Beveridge, D.L. (1970) Approximate molecular orbital theory. McGraw-Hill.
[22] Harker, A.H. and Larkins, F.P. (1979) Journal of Physics C: Solid State Physics, 12, 2497-2507. doi:10.1088/0022-3719/12/13/014

[23] Freund, H. and Hohlneicher, G. (1979) Theoretica chimica acta, 51, 145-162. doi:10.1007/BF00554098

[24] Schmid, E. and Brodbe, E. (1985) Canadian Journal of Chemistry, 63, 1365-1371. doi:10.1139/v85-233

[25] Vogl, P., Hjalmarsons, H.P. and Dow, J.d. (1983) Journal of Physics and Chemistry of Solids, 44, 365-378. doi:10.1016/0022-3697(83)90064-1

[26] Slater, J.C. and Koster, G.F. (1954) Physical Review, 94, 1498-1529. doi:10.1103/PhysRev.94.1498

[27] Pople, J.A. Santry, D.P. and Segal, G.A. (1965) Journal of Chemical Physics, 43, 129-S135. doi:10.1063/1.1701475

[28] Bredow, T., Evarestov, R.A. and Jug, K. (2000) Physica Status Solidi B, 222, 495-514. doi:10.1002/1521-3951(200011)222:2<495::AID-PSSB4 95>3.0.CO;2-5

[29] Rogan, J. and Lagos, M. (2001) Philosophical Magazine $B, \mathbf{8 1}, 551-559$. doi:10.1080/13642810108225450

[30] Chadi, D.J. and Cohen, M.L. (1973) Physical Review B, 8, 5747-5753. doi:10.1103/PhysRevB.8.5747

[31] Janesko, B.G. and Yaron, D. (2004) Journal of Chemical Physics, 121, 5635-5645. doi:10.1063/1.1785771

[32] Daudal, R., Leroy, G., Peeters, D. and Sana, M. (1983) Quantum chemistry. John Wiley and Sons.

[33] Hassan, I.Z. (2001) Semiempirical self-consistent field calculations of III-V zinc-blende semiconductors. Ph. D. thesis. University of Al-Nahrain.

[34] Murnaghan, F.D. (1944) Proceedings of the National Academy of Science, 30, 244. doi:10.1073/pnas.30.9.244

[35] Zheng, J.C., Huan, C.H.A., Wee, A.T.S., Wang, R.Z. and Zheng, Y.M. (1999) Journal of Physics: Condensed Matter, 11, 927-935. doi:10.1088/0953-8984/11/3/030

[36] Abdulsattar, M.A. (1997) Self consistent field calculations of covalent semiconductors. Ph.D. Thesis, University of Baghdad.

[37] Allen, C. (1976) Astrophysical quantities. Athlone Press.

[38] Lee, S.H., Kang, J.H. and Kang, M.H.J. (1997) The Korean Physical Society, 31, 811-814.

[39] Omer, M.S. (2007) Materials Research Bulletin, 42, 319-326. doi:10.1016/j.materresbull.2006.05.031

[40] Kumar, V. and Sastry, B.S.R. (2001) Crystal Research and Technology, 36, 565-569. doi:10.1002/1521-4079(200107)36:6<565::AID-CRAT5 65>3.0.CO;2-F

[41] Lam, P.K., Cohen, M.L. and Martinez, G. (1987) Physical Review B, 35, 9190-9194. doi:10.1103/PhysRevB.35.9190

[42] Ferhat, M., Bouhafs, B., Zaoui, A. and Aourag, H. (1998) Journal of Physics: Condensed Matter, 10, 7995-8006. doi:10.1088/0953-8984/10/36/010

[43] Wang, S.Q. and Ye, H.Q. (2002) Physical Review B, 66, 235111/1-7.

[44] Schlosser, H. and Ferrante, J. (1988) Physical Review B, Condensed Matter, 37, 4351-4357.

[45] Soma, T., Sawaoka, S. and Saito, S. (1974) Materials Research Bulletin, 9, 755. doi:10.1016/0025-5408(74)90110-X

[46] Calzaferri, G. and Rytz, R. (1996) Journal of Physical Chemistry, 100, 11122-11124. doi:10.1021/jp960840t

[47] Levitas, V.I. and Shvedov, L.K. (2002) Physical Review 
$B, \mathbf{6 5}, 104109 / 1-6$.

[48] Merdan, M.G. (2005) Self-consistent field calculations of the effect of pressure and temperature on some properties of grey tin crystal. Master's Thesis, Babylon University.

[49] Sekkal, W., Bouhaf, B., Aourag, H. and Certier, M. (1998) Journal of Physics: Condensed Matter, 10, 4975-4984. doi:10.1021/ip960840t

[50] Mujica, A., Rubio, A., Munoz, A. and Need, R.J. (2003) Reviews of Modern Physics, 75, 863. doi:10.1103/RevModPhys.75.863

[51] Jayawardane, D.N., Pickard, C.J., Brown, L.M. and Payne, M.C. (2001) Physical Review B, 64, 11517/1-4.

[52] Reddy, R.R., Ahammed, Y.N., Abdul Azeem, P., Gopal, K.R., Devi, B.S. and Rao, T.V.R. (2003) Defence Science Journal, 53, 239-248.

[53] Murrel, J.N., Kettle, S.F.A. and Tedder, J.M. (1982) The chemical bond. 2nd Edition, Translated to Arabic by M. N. Al-Zakum, University of Basrah.

[54] Ladd, M.F.C. (1983) Structure and bonding in solid state chemistry. Translated to Arabic by I. J. Selomi, University of Mosul.

[55] Adamska, C.M., Sloma, P. and Tomaszeewski, J. (2006) Journal of Physics: Condensed Matter, 18, 751-758. doi:10.1088/0953-8984/18/2/028

[56] Sibona, G.J. Schreiber, S., Hoppe, R.H.W., Strizker, B. and Revnic, A. (2003) Materials Science in Semiconductor Processing, 6, 71-76. doi:10.1016/S1369-8001(03)00073-8

[57] Phillips, J.C. (1956) Physical Review, 104, 1263-1277.

[58] Kittel, C. (1996) Introduction to solid state physics. 7th Edition, John Wiley and Sons. doi:10.1103/PhysRev.104.1263

[59] Glazov, V.M. and Shchelikov, O.D. (1998) Semiconductor Equipment and Materials International, 32, 382-384.

[60] Olguín, D., Cantarero, A., Ulrich, C. and Syassen, K. (2003) Physica Status Solidi B, 235, 456-463. doi:10.1002/pssb.200301602

[61] Audronis, M., Valiulis, A.V. and Silickas, P. (2004) Journal of Materials Science, 10, 152-156.

[62] Jiang, J.Z., Lindelov, H., Gerward, L., Ståhl, K., Recio, J. M., Mori-Sanchez, P., Carlson, S., Mezouar, M., Dooryhee, E., Fitch, A. and Frost, D.J. (2002) Physical Review $B, \mathbf{6 5}, 161202 / 1-4$.

[63] D’Evelyn, M.P. and Taniguchi, T. (1998) Elastic properties of translucent polycrystalline cubic boron nitride as characterized by the dynamic resonance method. GE Research \& Development Center, General Electric Company.

[64] Wang, B.T., Zhang, P., Shi, H.L., Sun, B. and Li, W.D. (2010) The European Physical Journal B, 74, 303-308. doi:10.1140/epjb/e2010-00081-x

[65] Hung, V.V., Jindo, K.M. and Haun, P.T.M. (2006) Journal of Physics: Condensed Matter, 18, 283-293. doi:10.1088/0953-8984/18/1/021

[66] Mijbil, Z.Y. (2011) Journal of Bab. University, 19, Under Publishing.

[67] Garai, J. and Laugier, A. (2007) Journal of Applied Physics, 101, 023514/1-4.

[68] Anderson, O.L. (1966) Physical Review, 144, 553-557. doi:10.1103/PhysRev.144.553

[69] Nandanpawar, M.L. and Rajagopalan, S. (1978) Journal of Applied Physics, 49, 3976-3979. doi: $10.1063 / 1.325408$

[70] Wachtman, J.B. Jr., Tefft, W.E., Lam, D.G., Jr., and Apstein, C.S. (1961) Physical Review, 122, 1754-1759. doi:10.1103/PhysRev.122.1754

[71] Sanjurjo, J.A., López-Cruz, E., Vogl, P. and Cardona, M. (1983) Physical Review B, 28, 4579-4584. doi:10.1103/PhysRevB.28.4579

[72] Trommer, R., Müller, H., Cardona, M. and Vogl, P. (1980) Physical Review B, 21, 4869-4878. doi:10.1103/PhysRevB.21.4869

[73] Burstein, E., Perkowitz, S. and Brodsky, M.H. (1968) Journal de Physique, 29, C4/78-83.

[74] Miranda, L.C.M. and Haar, ter D. (1972) Revista Brasileira de Ensino de Física, 2, 77-86.

[75] Molinàs-Mata, P.A., Shields, J. and Cardona, M. (1993) Physical Review B, 47, 1866-1875. doi:10.1103/PhysRevB.47.1866

[76] Raj, G.D. (2004) Solid state physics. 1st Edition, Anmol Pub. Pvt. Ltd..

[77] Xu, C.H., Wang, C.Z., Chan, C.T. and Ho, K.M. (1991) Physical Review B, 43, 5024-5027. doi:10.1103/PhysRevB.43.5024

[78] Kleinman, L. and Phillips, J.C. (1960) Physical Review, 117, 460-464. doi:10.1103/PhysRev.117.460

[79] Yang, D., Su, Z., Du, Y., Ji, X., Yang, X., Gong, X. and Zhang, T. (2006) Chinese Physics Letters, 23, 13241326. doi:10.1088/0256-307X/23/5/072

[80] Dovesi, R., Pisani, C., Roetti, C. and Dellarole, P. (1981) Physical Review B, 24, 4170-4176. doi:10.1103/PhysRevB.24.4170

[81] Chadi, D.J. (1977) Physical Review B, 16, 3572-3578. doi:10.1103/PhysRevB.16.3572

[82] Mori, Y., Ikai, T., Teranishi, R. and Takarabe, K. (2003) Physica Status Solidi B, 235, 302-306. doi:10.1002/pssb.200301573

[83] Kim, K., Lambrecht, W.R.L. and Segall, B. (1996) Physical Review B, 53, 16310-16326. doi:10.1103/PhysRevB.53.16310

[84] Jain, S.C., Willander, M. and Maes, H. (1996) Semiconductor Science and Technology, 11, 641-671. doi:10.1088/0268-1242/11/5/004

[85] Vurgaftmana, I., Meyer, J.R. and Mohan, L.R.R. (2001) Journal of Applied Physics, 89, 5815-5875. doi:10.1063/1.1368156

[86] Pässler, R. (1999) Physica Status Solidi B, 216, 975-1007. doi:10.1002/(SICI)1521-3951(199912)216:2<975::AIDPSSB975>3.0.CO;2-N

[87] Olguín, D., Cantarero, A. and Cardona, M. (2000) Physica Status Solidi B, 220, 33-39. doi:10.1002/1521-3951(200007)220:1<33::AID-PSSB33 $>3.0 . \mathrm{CO} ; 2-\mathrm{J}$

[88] Fan, H.Y. (1951) Physical Review, 82, 900-905. doi:10.1103/PhysRev.82.900

[89] Raczkowski, D., Canning, A. and Wang, L.W. (2001) Physical Review B, 46, 121101/1-4.

[90] Cotton, F.A. and Wilkinson, G. (1976) Basic inorganic chemistry. John Wiley \& Sons.

[91] Rose, J., Smith, J.R., Guinea, F. and Ferrante, J. (1984) Physical Review B, 29, 2963-2969. doi:10.1103/PhysRevB.29.2963

[92] Sánchez, P.M., Pendás, A.M. and Luaña, V. (2002) Journal of the American Chemical Society, 124, 14721- 
14723. doi:10.1021/ja027708t

[93] Bouhafs, B., Aourag, H. and Certier, M. (2000) Journal of Physics: Condensed Matter, 12, 5655-5668. doi:10.1088/0953-8984/12/26/312

[94] Wu, R.Q., Peng, G.W., Lui, L. and Feng, Y.P. (2006)
Journal of Physics: Condensed Matter, 18, 569-575. doi:10.1088/0953-8984/18/2/015

[95] Al-Jelawy, I.O.R. (2005) Effect of pressure and temperature on some properties of diamond crystal using hartree-fock method. Master Thesis, University of Babylon. 